\title{
Increased Prevalence of Developmental Venous Anomalies in Children with Intracranial Neoplasms
}

\author{
B.V. Jones, L. Linscott, G. Koberlein, T.R. Hummel, and J.L. Leach
}

\begin{abstract}
BACKGROUND AND PURPOSE: Developmental venous anomalies are considered variants of venous development that, in and of themselves, are of little clinical import. A possible association between intrinsic brain tumors and developmental venous anomalies has been suggested, but a rigorous investigation has not been performed. The aim of this study was to assess any association between developmental venous anomalies and intrinsic brain neoplasms.
\end{abstract}

MATERIALS AND METHODS: A radiology report text search of terms used to describe developmental venous anomalies was performed on a study population of 580 patients with primary intracranial neoplasms and on a control population of 580 patients without neoplasms from the same time period. All positive results were reviewed to confirm that the report was describing a developmental venous anomaly, and the imaging examination was reviewed to confirm the diagnosis.

RESULTS: Fifty-nine of the 580 subjects with brain tumors (10.17\%) had a developmental venous anomaly identified by report and confirmed on review of the imaging. Thirty-one of the 580 controls (5.34\%) had a developmental venous anomaly identified by report and confirmed on review of the imaging $(P=.003)$. No statistically significant difference was noted in the prevalence of developmental venous anomalies among tumor types. No developmental venous anomaly drained the vascular territory of the tumor, and there was no correlation between the location of the developmental venous anomaly and the location of the neoplasm.

CONCLUSIONS: The prevalence of developmental venous anomalies in this pediatric population with intracranial primary neoplasms is significantly greater than in those without neoplasms, suggesting an association that may be related to shared causative factors or susceptibilities to the development of these 2 separate entities.

ABBREVIATION: DVA = developmental venous anomaly

D evelopmental venous anomalies (DVAs) are vascular lesions that consist of a group of venous channels that drain into a common collecting vein, which, in turn, traverses the brain parenchyma to drain to either the superficial or deep venous systems. They are the most common cerebral vascular malformation, frequently identified on routine MR imaging of the brain with contrast. Postmortem studies 3 decades ago suggested a prevalence of $2.7 \%{ }^{1}$; however, with the use of modern imaging techniques, the prevalence is estimated to be much higher $(6.4 \%) .^{2}$ DVAs are typically considered variants of venous devel-

Received January 30, 2015; accepted after revision February 5.

From the Department of Radiology (B.V.J., L.L., G.K., J.L.L.) and Division of Pediatric Oncology (T.R.H.), Cincinnati Children's Hospital Medical Center, University of Cincinnati College of Medicine, Cincinnati, Ohio.

Please address correspondence to Blaise V. Jones, MD, Cincinnati Children's Hospital Medical Center, 3333 Burnet Ave, ML 5031, Cincinnati, OH 45229;

e-mail: blaise.jones@cchmc.org; @CincyKidsRad

http://dx.doi.org/10.3174/ajnr.A4352 opment that, in and of themselves, are of little clinical import. However, a small percentage of DVAs have been associated with such findings as cavernous malformations, ${ }^{3,4}$ thrombosis with subsequent venous infarction, ${ }^{5,6}$ lobar atrophy, ${ }^{7}$ T2 and FLAIR signal-intensity abnormalities, ${ }^{8,9}$ perfusion abnormalities, ${ }^{10,11}$ and SWI hypointensities. ${ }^{12}$

In our daily pediatric neuroradiology practice, we had noticed a high prevalence of presumed incidental DVAs in our oncology patient population. A possible association between intrinsic brain tumors and DVAs had been suggested before by Pryor et al, ${ }^{13}$ but a rigorous investigation has not been performed. This study tested the null hypothesis that there is no association between DVAs and intrinsic brain neoplasms.

\section{MATERIALS AND METHODS}

This retrospective study was approved by our institutional review board. The study population was derived from a data base of patients referred to the neuro-oncology service at our institution 
Table 1: Study and control population characteristics ${ }^{\mathrm{a}}$

\begin{tabular}{|c|c|c|c|c|c|c|c|}
\hline & \multirow[b]{2}{*}{ Mean Age (Range) } & \multicolumn{4}{|c|}{ Field } & \multicolumn{2}{|c|}{ Scan Type } \\
\hline & & Male & Female & $1.5 \mathrm{~T}$ & $3 T$ & Planar & Volumetric \\
\hline Neoplasm & $9.1 \mathrm{yr}$ (1 day to $35 \mathrm{yr}$ ) & 306 & 274 & 504 & 76 & 470 & 110 \\
\hline Controls & $10.2 \mathrm{yr}$ (1 day to $31 \mathrm{yr}$ ) & 274 & 306 & 486 & 94 & 473 & 107 \\
\hline
\end{tabular}

a The neoplasm group was slightly younger than the control group ( $P=.001$ by 2-tailed $t$ test). No significant differences were identified in sex $(P=.07)$, field strength $(P=.16)$, or scan type $(P=.89)$ between the 2 groups.

tification, $\sim 10 \%$ of the reported negative cases from each group (55 from the study population and 55 from the control population) were randomly selected and analyzed for the presence of DVAs.

\section{Statistical Analysis}

Statistical analysis of frequency data was

Table 2: Prevalence of DVAs in study and control populations ${ }^{a}$

\begin{tabular}{lccccc}
\hline & Total & DVA+ (\%) & 95\% Cl & Z Score & $\boldsymbol{P}$ Value \\
\hline Neoplasm & 580 & $59(10.17)$ & $7.97-12.90$ & 3.07 & .003 \\
Controls & 580 & $31(5.34)$ & $3.79-7.48$ & & \\
\hline
\end{tabular}

Note:-DVA+ indicates the number of subjects in group who had a confirmed DVA. a The neoplasm group had a $>10 \%$ prevalence of DVAs, compared with just over $5 \%$ for the control group. The latter is similar to recently published prevalence data.

from 1990 through 2011, a total of 719 cases. Cases were excluded from consideration if the diagnosis of neoplasm was not confirmed by either biopsy or documented progression; if the neoplasm was not intra-axial in origin; or if there was a vascular malformation (arteriovenous malformation, arteriovenous fistula, facial venous malformation), venous thrombosis, syndrome associated with vascular anomalies, major structural abnormalities limiting evaluation of the brain parenchyma, or no reviewable imaging. Metastatic neoplasms to the brain were also excluded. This process left a study population of $580 \mathrm{pa}-$ tients with primary intracranial neoplasms. A radiology report text search was then performed with the software program Softek Illuminate (Softek Solutions, Prairie Village, Kansas), by using the search terms "venous," "DVA," and "angioma." Positive results were reviewed to identify reports describing a DVA, and the imaging for each identified case was reviewed to confirm the diagnosis, document adequate diagnostic quality, and determine the location of the DVA. The MR imaging field strength $(1.5$ or $3 \mathrm{~T})$, whether postcontrast imaging was performed with a planar or volumetric technique, the year of the earliest available examination demonstrating the DVA, and patient age and sex were documented in all cases.

A control population of 580 studies was then derived from the radiology data base of all brain MR imaging examinations performed with intravenous contrast during the same study period. The number of control cases per year was matched to those of the study population, and the same text search was performed. Cases were excluded from consideration if there was a diagnosis of intracranial neoplasm or potential neoplasm (including nonspecific signal abnormalities) requiring follow-up. As with the tumor group, cases were also excluded if there was a vascular malformation (arteriovenous malformation, arteriovenous fistula, facial venous malformation), venous thrombosis, a syndrome associated with vascular anomalies, or major structural abnormalities limiting the evaluation of the brain parenchyma. The same test search was performed on this control population, positive results were reviewed to confirm that the report was describing a DVA, and the imaging examination was reviewed to confirm the diagnosis and document adequate diagnostic quality, with the same documentation of field strength, postcontrast technique, and subject demographics performed.

As a means of identifying false-negative rates of DVA iden- performed by using the $\chi^{2}$ test (continuity-corrected), confidence intervals of proportions (continuity-corrected), and z-ratio of independent proportions (VassarStats: Website for Statistical Computation; vassarstats.net). Continuous variables (age) were assessed with a 2 -tailed $t$ test (vassarstats.net). $P<.05$ was significant.

\section{RESULTS}

Our selection process resulted in 2 groups of subjects with MR imaging of the brain ( 1 without and 1 with brain tumors), evaluated during the same time period, by the same group of radiologists, using similar imaging techniques. Exclusion criteria were identical in each group, with the exception of the presence of intracranial neoplasm in the tumor cohort. Demographics and MR imaging study type distribution for the study and control groups are provided in Table 1. The tumor group was slightly younger than the control group (mean, 9.0 years versus 10.1 years; $P=.002,2$-tailed $t$ test). No significant differences were noted in sex $(P=.07)$, field strength $(P=.16)$, or scan type $(P=.89)$ between groups.

Fifty-nine of the 580 subjects with brain tumors (10.17\%) had a DVA identified by report and confirmed on review of the imaging. Thirty-one of the 580 controls (5.34\%) had a DVA identified by report and confirmed on review of the imaging (Table 2, $P=$ $.003)$. Forty-two of the positive cases in the study population had glial tumors (71\%); 5 had glioneuronal tumors; 9, embryonal; 2 ependymal; and 1, germ cell (Fig 1). This prevalence was similar to the distribution of tumor types in the study population overall, with the result that there was no statistically significant difference in the prevalence of DVAs among tumor types. Thirty-eight subjects had DVAs in the frontal lobes, 11 in the parietal lobes, and 4 in the temporal lobes, with 4 in the posterior fossa, 2 in the occipital lobes, and 1 in the basal ganglia. Four subjects had more than 1 DVA. None of the DVAs drained the vascular territory of the tumor, and there was no correlation between the location of the DVA and supratentorial-versus-infratentorial compartments.

The DVA was visible on the oldest imaging study available for review in all cases. In 51 of the 59 DVA-positive cases, the original study diagnosing a neoplasm was available for review, and in all of these, the DVA was visible. In the remaining 8 cases, the oldest imaging study available for review was not the original diagnostic examination that identified the neoplasm. In 4 of these 8 cases, the report of the original examination was available, and it documented the presence of the DVA. Of the remaining 4 cases, in one, the oldest available study was performed 5 months after diagnosis, with no intervening treatment. In another, the oldest available study was performed 2 years after surgical resection of the neoplasm, without intervening adjuvant therapy. In the 2 remaining the location of the neoplasm in terms of lobe, side of the brain, or 


\section{Distribution of Tumor Types Without DVA}

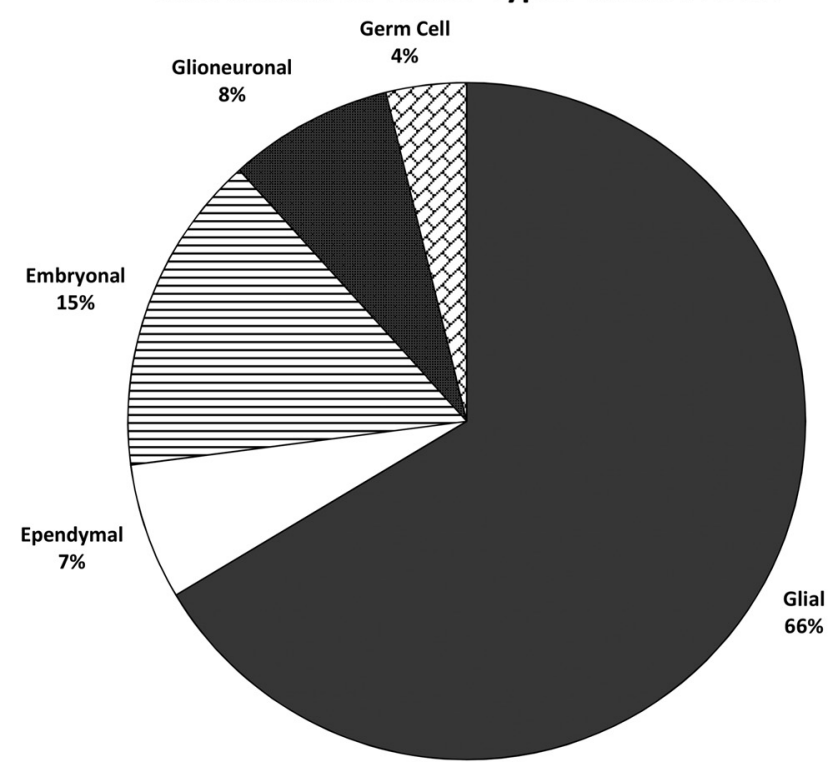

Distribution of Tumor Types With DVA

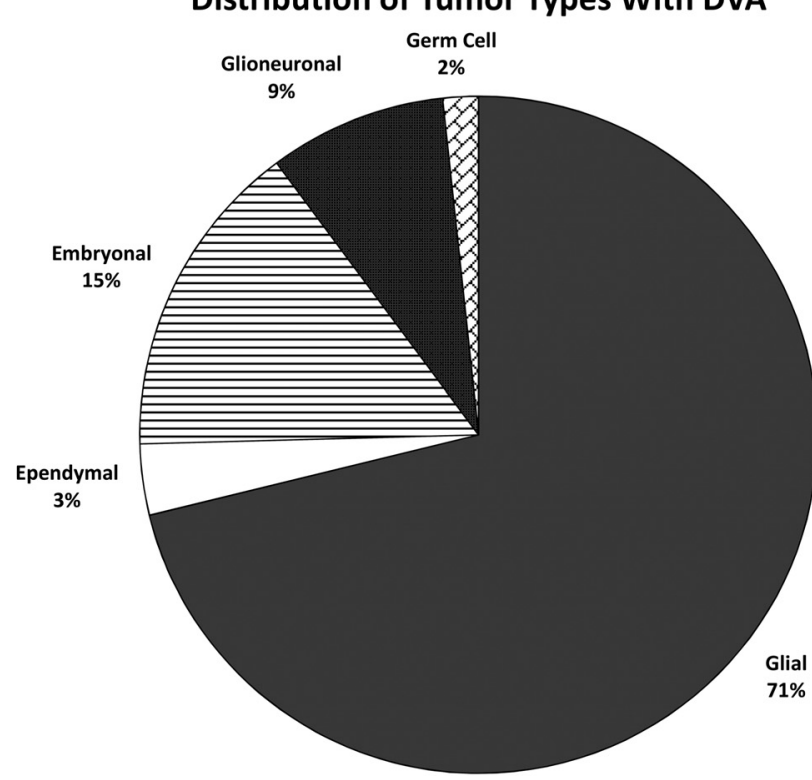

FIG 1. Pie charts show the distribution of tumor types among the study population in those without DVAs (left) and those with DVAs (right). Both groups are dominated by glial tumors, without a statistically significant difference between the 2 .

cases, the oldest examinations available for review were performed $>4$ years after diagnosis and treatment of the neoplasm, and there was intervening treatment with both chemotherapy and radiation therapy.

Of the 55 cases with negative findings randomly selected from the study population, one (1.8\%) showed a DVA on review. Similarly, one of the cases randomly selected from the control population demonstrated a DVA on review. Extrapolating this falsenegative rate to the entire population would suggest a prevalence of approximately $12 \%$ in children with neoplasms and $7 \%$ in those without.

\section{DISCUSSION}

DVAs are encountered with frequency in daily neuroradiology practice and are generally considered to be essentially benign variants of venous development. Several theories have been proposed to explain their etiology, the most broadly accepted of which is that they represent a response of the developing brain to a regional compromise of the normal venous drainage caused by stenosis, thrombosis, or maldevelopment. ${ }^{14}$ Others have suggested that abnormalities of fetal angiogenesis and regression may lead to their development. ${ }^{15}$ There is consensus that they arise in utero, though there have been reports documenting postnatal evolution. ${ }^{16}$

It has long been recognized that a substantial number of DVAs are associated with cavernous malformations, and it is theorized that both lesions represent differing responses to a compromise of regional venous drainage. ${ }^{17-20}$ However, DVAs are also seen with a high prevalence in patients with lymphatic or venolymphatic malformations of the head and neck, ${ }^{21,22}$ and they have an increased incidence in a variety of genetically driven syndromes such as the blue rubber bleb nevus syndrome. ${ }^{23}$ These associations suggest that DVA development is not solely a reaction to focal abnormalities of venous drainage but may also be a consequence of widespread perturbations of vascular development. Re- cent reports have identified associated parenchymal signal abnormalities in $11.6 \%$ of children and young adults ${ }^{9}$ and in $12.5 \%$ of older adults ${ }^{8}$ with DVAs, suggesting that the altered drainage they provide may have consequences on the surrounding brain. All of these associations lend credence to concerns that DVAs may have a deleterious impact on the surrounding brain or may be a harbinger of more widespread abnormalities. Our findings indicate that DVAs may be more prevalent in pediatric patients with intracranial neoplasms than in controls.

Because DVAs are lesions that primarily impact the brain parenchyma, we limited our study population to those patients with intra-axial tumors. The distribution of tumor types in our study population parallels the known relative prevalence of these lesions in the pediatric population, and the prevalence of DVAs within our study subjects generally follows the distribution of tumor types. We found no statistically significant association of DVA prevalence with tumor type, and the distribution of DVA location within the brain was consistent with that in prior reports. ${ }^{24}$

The reason that DVAs may be more prevalent in children with primary intracranial neoplasms is not known. The fact that in none of our cases did the DVA drain the territory of the neoplasm essentially eliminates any direct causative link. However, if DVAs are exclusively the result of focal compromise of venous development, the same abnormality that led to the venous compromise may have caused a concomitant insult elsewhere in the brain that increases the risk of developing a neoplasm. Alternatively, if they are a consequence of a more widespread alteration of vascular development, as suggested by their association with venolymphatic malformations and neurocutaneous syndromes, it is conceivable that they may be associated with an increased vulnerability to neoplastic differentiation in the brain tissue.

It is reasonable to question whether the DVAs in this population could be a consequence of treatment for neoplasms, much as cavernous malformations are associated with prior radiation 
therapy. ${ }^{25}$ However, in all except 2 of our cases, there was the clearly documented presence of the DVA before any chemotherapy or radiation treatment. There was no instance of a DVA becoming evident with time; they could be identified on the oldest available study for review in all cases. Furthermore, growth in response to therapy contradicts the generally accepted developmental nature of DVAs and would suggest that the DVAs identified in this analysis are substantially different from those recognized in the general population. The standard distribution and characteristic appearance of the DVAs in our study population would argue against this hypothesis. The possibility that the development of a DVA is somehow caused by the presence of a neoplasm elsewhere in the brain cannot be excluded, but the possible causal link is not apparent.

This study is weakened by its retrospective nature and dependence on reporting. It is not a study of lesion prevalence but rather one of lesion-identification prevalence, raising the question of bias in the interpretation of studies in controls compared with subjects. However, the prevalence of DVAs identified in our control population was similar to that demonstrated in the most recent imaging-based studies, suggesting a similar degree of sensitivity of detection. Furthermore, a 10\% sampling of cases with negative findings from each group demonstrated no difference between detection rates. The same group of fellowship-trained neuroradiologists generated the reports in each group during their daily clinical practice; because they were working under the general assumption that DVAs are incidental variants with no relation to neoplasms, it is unlikely that they would have been sensitized to their presence in oncologic patients relative to controls. We found no significant differences in examination techniques that would favor the detection of DVA in one group or the other. The fact that most of the subjects had multiple examinations undoubtedly increased the chance of lesion detection, but nearly all DVAs were recognized on the initial studies performed, minimizing the impact of multiple studies.

\section{CONCLUSIONS}

We found that the prevalence of DVAs in children with primary intracranial neoplasms is significantly greater than in those without neoplasm, suggesting an association that may be related to shared causative factors or susceptibilities to development of these 2 separate entities. A prospective study could more clearly delineate the association between these processes and provide greater insight into the potential causative factors. New knowledge gained about either entity should be considered in the study of the other, and one should recognize that there may be more commonality among these lesions than previously appreciated.

\section{REFERENCES}

1. Sarwar M, McCormick WF. Intracerebral venous angioma: case report and review. Arch Neurol 1978;35:323-25

2. Gökçe E, Acu B, Beyhan M, et al. Magnetic resonance imaging findings of developmental venous anomalies. Clin Neuroradiol 2014;24:135-43

3. Abe T, Singer RJ, Marks MP, et al. Coexistence of occult vascular malformations and developmental venous anomalies in the cen- tral nervous system: MR evaluation. AJNR Am J Neuroradiol 1998;19:51-57

4. Beall DP, Bell JP, Webb JR, et al. Developmental venous anomalies and cavernous angiomas: a review of the concurrence, imaging, and treatment of these vascular malformations. J Okla State Med Assoc 2005;98:535-38

5. Kiroglu Y, Oran I, Dalbasti T, et al. Thrombosis of a drainage vein in developmental venous anomaly (DVA) leading venous infarction: a case report and review of the literature. J Neuroimaging 2011;21:197-201

6. Teo M, St George J, Jenkins S, et al. Developmental venous anomaliestwo cases with venous thrombosis. Br J Neurosurg 2012;26:886-87

7. San Millán Ruíz D, Delavelle J, Yilmaz H, et al. Parenchymal abnormalities associated with developmental venous anomalies. Neuroradiology 2007;49:987-95

8. Santucci GM, Leach JL, Ying J, et al. Brain parenchymal signal abnormalities associated with developmental venous anomalies: detailed MR imaging assessment. AJNR Am J Neuroradiol 2008;29:1317-23

9. Linscott LL, Leach JL, Zhang B, et al. Brain parenchymal signal abnormalities associated with developmental venous anomalies in children and young adults. AJNR Am J Neuroradiol 2014;35:1600-07

10. Iv M, Fischbein NJ, Zaharchuk G. Association of developmental venous anomalies with perfusion abnormalities on arterial spin labeling and bolus perfusion-weighted imaging. J Neuroimaging 2015;25:243-50

11. Sharma A, Zipfel GJ, Hildebolt C, et al. Hemodynamic effects of developmental venous anomalies with and without cavernous malformations. AJNR Am J Neuroradiol 2013;34:1746-51

12. Takasugi M, Fujii S, Shinohara Y, et al. Parenchymal hypointense foci associated with developmental venous anomalies: evaluation by phase-sensitive MR imaging at 3T. AJNR Am J Neuroradiol 2013;34:1940-44

13. Pryor J, Setton A, Berenstein A. Venous anomalies and associated lesions. Neurosurg Clin N Am 1999;10:519-25

14. Saito Y, Kobayashi N. Cerebral venous angiomas: clinical evaluation and possible etiology. Radiology 1981;139:87-94

15. Wilson CB. Cryptic vascular malformations. Clin Neurosurg 1992;38:49-84

16. Leach JL, Howard T, Abruzzo T, et al. Postnatal evolution of developmental venous anomaly. J Pediatr Neuroradiol 2012;1:305-11

17. Campeau NG, Lane JI. De novo development of a lesion with the appearance of a cavernous malformation adjacent to an existing developmental venous anomaly. AJNR Am J Neuroradiol 2005;26:156-59

18. Maeder P, Gudinchet F, Meuli R, et al. Development of a cavernous malformation of the brain. AJNR Am J Neuroradiol 1998;19:1141-43

19. Dillon WP. Cryptic vascular malformations: controversies in terminology, diagnosis, pathophysiology, and treatment. AJNR Am J Neuroradiol 1997;18:1839-46

20. Cakirer S. De novo formation of a cavernous malformation of the brain in the presence of a developmental venous anomaly. $\mathrm{Clin} \mathrm{Ra}$ diol 2003;58:251-56

21. Boukobza M, Enjolras O, Guichard JP, et al. Cerebral developmental venous anomalies associated with head and neck venous malformations. AJNR Am J Neuroradiol 1996;17:987-94

22. Bisdorff A, Mulliken JB, Carrico J, et al. Intracranial vascular anomalies in patients with periorbital lymphatic and lymphaticovenous malformations. AJNR Am J Neuroradiol 2007;28:335-41

23. Gabikian P, Clatterbuck RE, Gailloud P, et al. Developmental venous anomalies and sinus pericranii in the blue rubber-bleb nevus syndrome: case report. J Neurosurg 2003;99:409-11

24. Lee C, Pennington MA, Kenney CM 3rd. MR evaluation of developmental venous anomalies: medullary venous anatomy of venous angiomas. AJNR Am J Neuroradiol 1996;17:61-70

25. Nimjee SM, Powers CJ, Bulsara KR. Review of the literature on de novo formation of cavernous malformations of the central nervous system after radiation therapy. Neurosurg Focus 2006;21:e4 